\title{
Large-scale cultivation of Caenorhabditis elegans in a bioreactor using a labor-friendly fed-batch approach
}

\author{
Ruud Heshof', Bram Visscher', Simone Prömel ${ }^{\ddagger, 2}$ \& Samantha Hughes*, ${ }^{\star, 1}$
}

\begin{abstract}
Caenorhabditis elegans is an invertebrate model organism used in many areas of biology including developmental biology and the identification of molecular mechanisms and pathways. However, several experimental approaches require large quantities of worms, which is limiting and timeconsuming. We present a protocol that uses modern fermentation methodology to effectively produce large numbers of $C$. elegans using a 7-I bioreactor in a fed-batch cultivation procedure. The production is modular and flexible as well as being a selfcontrolled system, thus not much labor is required until harvesting $C$. elegans. The high-yield worm cultivation is flexible and simple to amend, and now allows for the extended application of C. elegans as a model organism and expression system, including largescale protein production.
\end{abstract}

\section{METHOD SUMMARY}

Our method of producing large quantities of Caenorhabditis elegans using a bioreactor is a simple and flexible protocol. It is a fed-batch cultivation in which Escherichia coli is grown in its own bioreactor and serves as feed for $C$. elegans. The quantity of the $C$. elegans biomass can be easily titrated via the $E$. coli biomass.

\section{KEYWORDS}

bioreactor $\cdot$ Caenorhabditis elegans - liquid culturing $\cdot$ medium- to largescale production

'HAN BioCentre, HAN University of Applied Sciences, Nijmegen, 6525EM, The Netherlands; ${ }^{2}$ Rudolf Schönheimer Institute of Biochemistry, Leipzig University, Leipzig, 04103, Germany; *Author for correspondence: samantha.hughes@han.nl; ¥These authors contributed equally to the work

BioTechniques 67: 33-39 (July 2019) 10.2144/ btn-2019-0008

Vol. $67 \mid$ No. 1 (c) 2019 Samantha Hughes
The nematode Caenorhabditis elegans is a popular and widely used small invertebrate model organism for addressing many biological questions due to the numerous technical advantages it bears over other cellular and animal models. C. elegans is transparent, easy to cultivate and a vast toolkit exists for genomic manipulation and transgenesis, making $C$. elegans an ideal organism for many in vivo approaches. Thus, transfer of knowledge gained from biochemical analyses into in vivo studies is simple and can yield informative pictures of molecular mechanisms in the context of an entire organism. However, ex vivo biochemical analyses, for example for protein purification and characterization or unbiased screening for interaction partners, are difficult due to the small size of the worm and the resulting low amount of biomaterial. As the labor-intensive nature of sample preparation is the main limiting factor in many experiments, generating large numbers of nematodes in an easily manageable fashion is highly attractive. While it is generally possible to grow large amounts of $C$. elegans using vast numbers of petri dishes containing solid media, or liquid culture using flasks, meeting the demand to provide large numbers of healthy and well-fed $C$. elegans under reproducible standard conditions is only possible using bioreactors. Bioreactors are large closed vessels that are specifically designed to allow for the growth of cells or small organisms in a biologically and chemically defined and controllable liquid environment. Parameters such as aeration, temperature and $\mathrm{pH}$ are monitored and adjusted constantly, rendering optimized growth conditions. Furthermore, food or other substances can be added, either in a batch (once at the beginning), continuously (constantly with simultaneous withdrawal of equal volumes of culture medium) or as a fed batch (in batches upon concentration decrease without withdrawal of culture medium). To date, generic fermentation technologies designed for bioreactor cultivation of microorganisms have proven highly adaptable to new challenges and opportunities. Indeed, large-scale tissue culture of mammalian cells [1], plant cells [2] and fungi [3] have been established.

For $C$. elegans, only a few bioreactor protocols already exist [4-7]. However, these are rather labor intensive, mainly as the food, Escherichia coli, is harvested, centrifuged and then added to the $C$. elegans bioreactor (batch or fed-batch cultivation). However, large-scale $C$. elegans cultures with kilograms of worms have been achieved $[8,9]$. It is important to stress that these methods are for very largescale cultivation, at volumes over $100 \mathrm{I}$, which is not feasible for most laboratories, and these methods are extremely labor intensive. Furthermore, current protocols lack the detail concerning the exact bioreactor conditions to grow $C$. elegans in large numbers in liquid culture, which makes the process even more challenging. Producing large quantities of $C$. elegans is extensive and requires elaborate bioengineering to optimize. Dissolved oxygen availability $[10,11]$, temperature fluctuations and food availability are problematic as the worms may enter the dauer state, arrest or die if these are incorrect. In addition, the mechanics of the bioreactor are key to optimal worm growth. The sparger and impeller are essential for aeration control as well as agitation speed, which must be low enough to prevent shear of nematodes but sufficient to ensure correct oxygenation levels [11]. Here, we provide the bioreactor conditions for a simple, easily manageable and flexible protocol required to produce C. elegans nematodes.

\section{GENERAL CONSIDERATIONS OF WORKFLOW FOR PRODUCING LARGE QUANTITIES OF C. ELEGANS}

The general setup of this cultivation protocol to produce large quantities of $C$. elegans is - 


\section{Benchmarks}

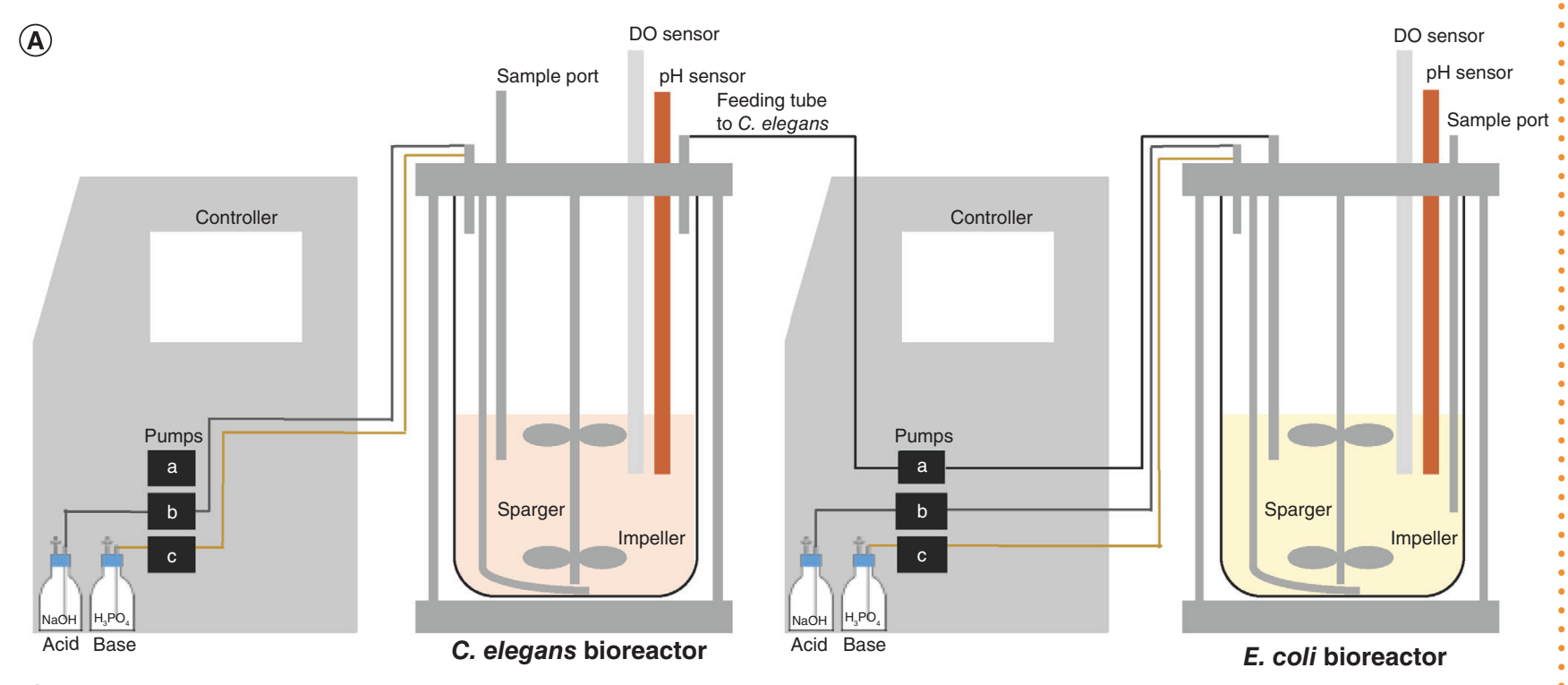

(B)

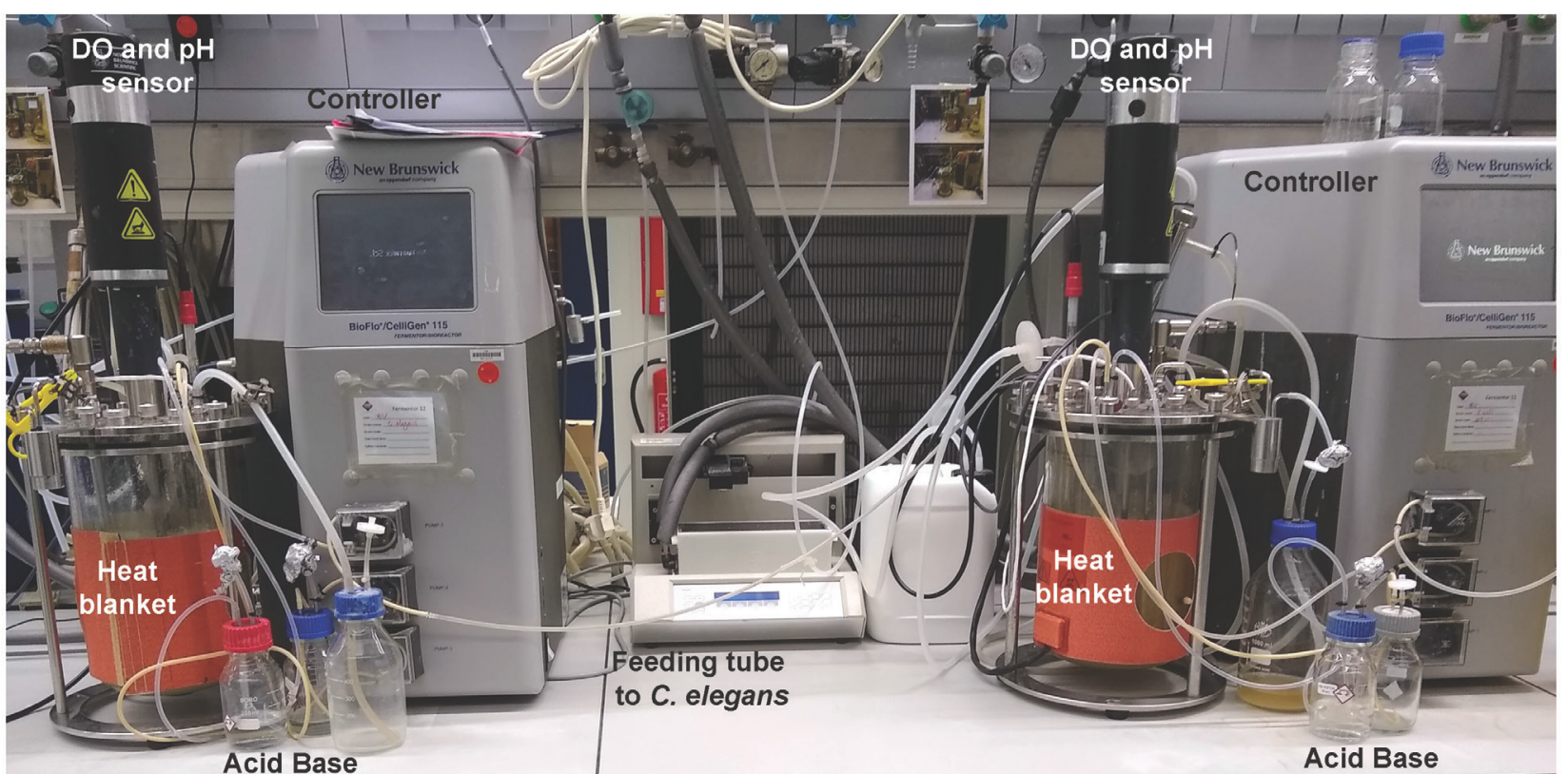

C. elegans bioreactor

E. coli bioreactor

Figure 1. Depiction of the overall set-up of the fed-batch cultivation for the medium- to large-scale production of Caenorhabditis elegans. This is a modern fermentation methodology to produce large biomass of $C$. elegans using a 7-I bioreactor. The production is modular and flexible, and done with two bioreactors, one to grow Escherichia coli $\mathrm{HB} 101$ as food source and one to grow C. elegans. Both bioreactors are connected and require two Rushton impellers and three pumps (black boxes). One pump is for the addition of feed (pump a), one to add the acid (pump b) and another for the addition of a base (pump c) to buffer the solutions. (A) Schematic diagram of the set-up for the two bioreactors. (B) Image of the set-up functioning in the laboratory. The size of each of the bioreactors is $27.7 \times 78.1 \mathrm{~cm}$ (diameter $\times$ height) while the size of the controllers is $24.7 \times 55.9 \times 62.9 \mathrm{~cm}(\mathrm{~W} \times \mathrm{D} \times \mathrm{H})$ each. DO: Dissolved oxygen.

- based on two bioreactors connected to each other (Figure 1). E. coli is first grown in one bioreactor and $C$. elegans cultivation is started subsequently using the bacteria from this as a food source. In this way, no intermediate steps such as centrifugation, washing and concentration of the $E$. coli are required, consequently the process is labor friendly. Furthermore, the entire cultivation process is automated and standardized, with minimal risk of contamination. The growth time for the $C$. elegans is approximately 1 week but can be altered depending on the desired biomass yield. The entire work process including time for preparation and $E$. coli growth adds up to 10-14 days. Furthermore, if only one bioreactor is available, this can be used to first produce $E$. coli and subsequently $C$. elegans. A general overview of the procedure and the time frame is depicted in Figure 2. 
(1) Escherichia coli

Starting plate

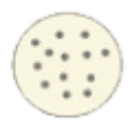

E. coli on LB plate $24 \mathrm{~h}$
Pre-culture

Single colony E. coli

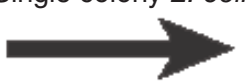

E. coli in $300 \mathrm{ml}$ LB broth

$24 \mathrm{~h}, 37^{\circ} \mathrm{C}, 150 \mathrm{rpm}$

Add entire $300 \mathrm{ml}$

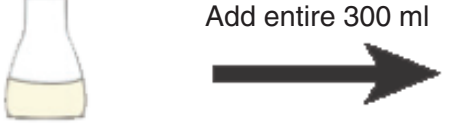

(2) Caenorhabditis elegans

Starting plate

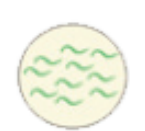

30 L4 stage animals

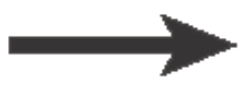

C. elegans on NGM plate grow to L4 stage
C. elegans in $500 \mathrm{ml} \mathrm{S-complete}$

4 days, $20^{\circ} \mathrm{C}, 150 \mathrm{rpm}$

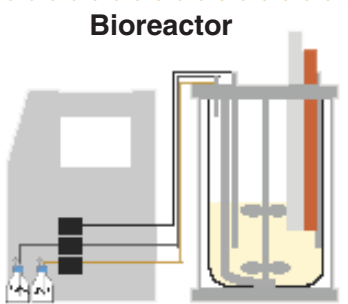

E. coli in 6 $\sim 8 \mathrm{~h}$

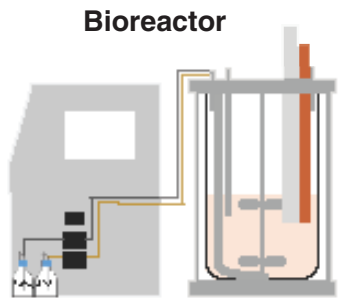

C. elegans in 11

Figure 2. A schematic diagram of the workflow and timeline. (1) First, an Escherichia coli HB101 culture must be made. A single colony is picked from a stock plate and used to inoculate $300 \mathrm{ml} \mathrm{LB}$ broth. After $24 \mathrm{~h}$, this is used to start an E. coli bioreactor of $6 \mathrm{I}$. (2) In parallel, a Caenorhabditis elegans pre-culture is prepared. Nematodes are grown to L4 stage on NGM and 30 worms are added to $500 \mathrm{ml} \mathrm{S-complete.} \mathrm{The} \mathrm{1-I} \mathrm{flasks} \mathrm{are} \mathrm{left} \mathrm{for} 4$ days at $20^{\circ} \mathrm{C}$, gently shaking at $150 \mathrm{rpm}$, at which point $100 \mathrm{ml}$ of the solution (containing approximately $21 \mathrm{worms} / \mathrm{ml}$ ) is added to $1 \mathrm{l}$ of S-complete in the $C$. elegans bioreactor.

The key advantage of this protocol is that as this is a self-controlled system, there is minimal labor requirement. To prepare the bioreactors takes approximately $4 \mathrm{~h}$, and $10 \mathrm{~min}$ per day is required to check the system and make any adjustments. The Biocommand software ensures that there is no need to manually check the feed levels. At the end of the process, approximately $4 \mathrm{~h}$ is required to clean the bioreactors.

\section{PREPARATION OF THE BIOREACTOR}

Before starting the cultivation process, one day of well-planned preparation is required to set up all reagents and solutions (Table 1).

\section{Bioreactor setup}

The bioreactors used in this study are 7-I flat-bottom vessels attached to a New Brunswick ${ }^{\mathrm{TM}}$ BioFlo 115 (Eppendorf, The Netherlands). Both bioreactors require a Rushton impeller at the bottom near a ringsparger and another in the middle, at around 3 I of volume. The sparger provides oxygen

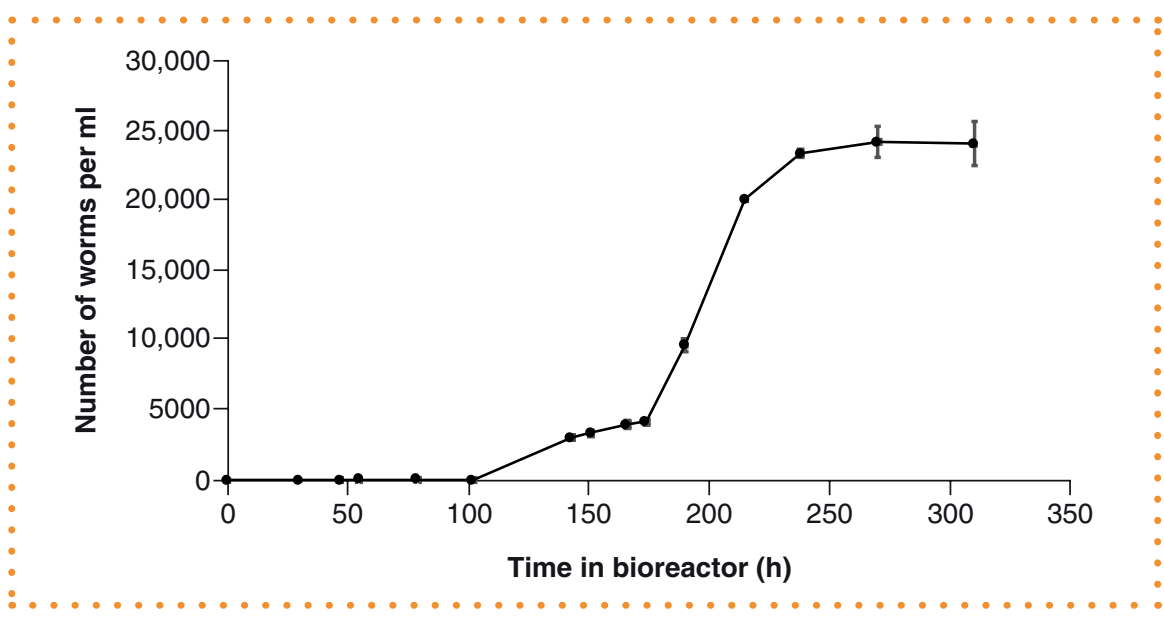

Figure 3. Increase in the number of worms over time in the bioreactor. The seed culture that was used to start the bioreactor had 21 worms $/ \mathrm{ml}$. Cholesterol was added at $71 \mathrm{~h}$ and $238 \mathrm{~h}$. A maximum of 24,000 worms $/ \mathrm{ml}$ was obtained. At each time point, the number of worms in the bioreactor was calculated as the average of 3-7 aliquots of $10 \mu \mathrm{l}$ taken from a $5-\mathrm{ml}$ sample from the bioreactor then scaled up to the volume in the bioreactor at that time point. Error bars are given as standard error of the mean from the counting of the aliquots from the bioreactor cultures.

to the system while the impeller rotates the solution inside the bioreactor (Figure 1A). Three pumps are needed: one for the feed addition, one to add the acid and a third to add the base (Figure 1A). The working area required is quite small (Figure $1 \mathrm{~B}$ ) and can be easily accommodated in any laboratory space. Specific parameters for each - - 


\section{Benchmarks}

Table 1. Solutions and buffers for Caenorhabditis elegans batch medium.

\begin{tabular}{|c|c|c|}
\hline Specific reagents and solutions & Supplier & Required for \\
\hline LB medium & Carl Roth & LB pre-culture \\
\hline Yeast extract & Gistex & Batch medium Nitrogen source \\
\hline BactoAgar & Formedium & NGM and LB plates \\
\hline Casein Peptone & Organo Technie & NGM \\
\hline Cholesterol ( $5 \mathrm{mg} / \mathrm{ml}$ in ethanol) & Sigma-Aldrich & NGM and S-complete \\
\hline Nystatin (20 mg/ml in DMSO) & Thermo Fisher Scientific & NGM and S-complete \\
\hline Penicillin/streptomycin $(10,000 \mathrm{U} / \mathrm{ml})$ & Lonza & S-complete \\
\hline Buffers and media: E. coli & Ingredients & Required volume \\
\hline Batch medium - carbon source & $5.45 \mathrm{~g} / \mathrm{l}$ glucose $\cdot \mathrm{H}_{2} \mathrm{O}$ & 5.51 \\
\hline Batch medium - nitrogen source & $\begin{array}{l}34.8 \mathrm{~g} / \mathrm{l} \text { yeast extract, } 6 \mathrm{~g} / \mathrm{l} \mathrm{NH}_{4} \mathrm{Cl} \\
16.8 \mathrm{~g} / \mathrm{l} \mathrm{MgSO} \\
\end{array}$ & $500 \mathrm{ml}$ \\
\hline Buffers and media: $C$. elegans & Ingredients & Required volume \\
\hline Phosphate buffer pH 6.0 & $108.3 \mathrm{~g} / \mathrm{I} \mathrm{KH}_{2} \mathrm{PO}_{4} 35.5 \mathrm{~g} / \mathrm{l} \mathrm{K}{ }_{2} \mathrm{HPO}_{4}$ & $25 \mathrm{ml}$ \\
\hline NGM & $\begin{array}{l}17 \mathrm{~g} / \mathrm{l} \text { BactoAgar, } 3 \mathrm{~g} / \mathrm{l} \mathrm{NaCl}, 2.5 \mathrm{~g} / \mathrm{l} \text { peptone } \\
\text { Sterilize by autoclaving, then add: } \\
1 \mathrm{ml} / \mathrm{l} 1 \mathrm{M} \mathrm{CaCl}, 1 \mathrm{ml} / \mathrm{l} \text { cholesterol, } \\
1 \mathrm{ml} / \mathrm{l} 1 \mathrm{M} \mathrm{MgSO}{ }_{4}, 25 \mathrm{ml} / \mathrm{l} \text { phosphate buffer }\end{array}$ & 11 \\
\hline Trace metal solution & $\begin{array}{l}1.86 \mathrm{~g} / \mathrm{l} \text { disodium EDTA, } \\
0.69 \mathrm{~g} / \mathrm{l} \mathrm{FeSO}{ }_{4} \cdot 7 \mathrm{H}_{2} \mathrm{O}, 0.2 \mathrm{~g} / \mathrm{l} \mathrm{MnCl} \cdot 4 \mathrm{H}_{2} \mathrm{O}, 0.29 \mathrm{~g} / \mathrm{l} \\
\mathrm{ZnSO}_{4} \cdot 7 \mathrm{H}_{2} \mathrm{O}, 0.025 \mathrm{~g} / \mathrm{l} \mathrm{CuSO}{ }_{4} \cdot 5 \mathrm{H}_{2} \mathrm{O} \\
\text { Sterilize by autocalving }\end{array}$ & 11 \\
\hline S-complete & 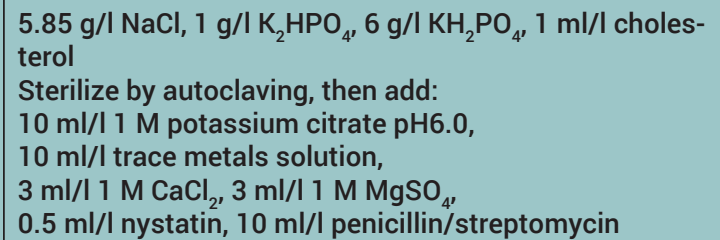 & 21 \\
\hline
\end{tabular}

All solutions and buffers are made with sterile $\mathrm{dH}_{2} \mathrm{O}$ unless indicated otherwise. To warrant sterility and to avoid any contaminations, all buffers and solutions are sterilized by autoclaving or filter sterilization, unless stated otherwise. Note that after sterilization by autoclaving, any additional components should be added via filter sterilization.

Table 2. Parameters used for the bioreactor cultivations of Escherichia coli and Caenorhabditis elegans.

\begin{tabular}{|l|l|l|}
\hline Parameter & E. coli & \multicolumn{2}{l|}{ C. elegans } \\
\hline Medium volume $(\mathrm{I})$ & 6 & 1 \\
\hline Agitation $(\mathrm{rpm})$ & $300-1200$ & 200 \\
\hline Dissolved oxygen control $(\%)$ & $20 \%-$ agitation cascade controlled & none \\
\hline Temperature $\left({ }^{\circ} \mathrm{C}\right)$ & 37 & 22 \\
\hline $\mathrm{pH}$ & $7.0 \pm 0.1$ & $6.5 \pm 0.1$ \\
\hline Acid & $6 \mathrm{M} \mathrm{H}_{3} \mathrm{PO}_{4}-$ pump speed $20 \%$ & $6 \mathrm{M} \mathrm{H}_{3} \mathrm{PO}_{4}-$ pump speed $20 \%$ \\
\hline Base & $6 \mathrm{M} \mathrm{NaOH}-$ pump speed $20 \%$ & $6 \mathrm{M} \mathrm{NaOH}-$ pump speed $20 \%$ \\
\hline Feed & None & E. coli when DO $\geq 20 \%$ \\
\hline Gas flow $(\mathrm{I} / \mathrm{min})$ & 0.7 & 2.0 \\
\hline
\end{tabular}

The bioreactors New Brunswick ${ }^{\mathrm{TM}}$ BioFlo 115 (Eppendorf, The Netherlands) with two Rushton impellers and three pumps. The parameters for both the E. coli and C. elegans bioreactor cultivations are shown. DO: Dissolved oxygen. 
- bioreactor cultivation are required to control the dissolved oxygen, $\mathrm{pH}$ and temperature (Table 2).

\section{Dissolved oxygen}

The dissolved oxygen (DO) sensor is calibrated to a range of $0-100 \%$. To do this, the bioreactor with all components, except the biomass, is made anaerobic using nitrogen gas. When this has stabilized, the $\mathrm{DO}$ sensor is set to a value of $0 \%$. The bioreactor is then made aerobic using air flow and set to $100 \%$ when stabilized.

$\mathrm{pH}$

The $\mathrm{pH}$ is controlled via a controller with a dead-band of 0.1 at $20 \%$ pump speed. Whenever the $\mathrm{pH}$ is \pm 0.1 from the set point, the pump will be turned on to add acid or base accordingly. The $20 \%$ pump speed is chosen so that the system has time to mix the acid or base properly, before being added again.

\section{Temperature}

The temperature is controlled via an external heat blanket and an internal cooling loop in which water circulates at $4^{\circ} \mathrm{C}$. Heating or cooling is monitored by the controller with the internal proportional-integral-derivative algorithm at factory settings. The temperature of the bioreactor is stable between 8 and $45^{\circ} \mathrm{C}$

\section{Feeding}

Feeding is controlled via a script using the Biocommand software. As soon as the DO is higher than $20 \%$ (e.g., the culture is not using oxygen since there is no food to oxidize for energy), the pump will be turned on. This will provide the $C$. elegans culture with $E$. coli. As soon as the DO drops to $20 \%$ or less (e.g., when the oxygen is being used as $E$. coli is present), the pump will be turned off. The DO will rise until a DO greater than $20 \%$ is reached, and the system is turned on again. In this way enough $E$. coli is always available for C. elegans as a food source.

\section{E. coli cultivation}

In these experiments, the E. coli strain HB101 is used as the food source for C. elegans. HB101 is a uracil auxotroph and resistant to penicillin and streptomycin. $\mathrm{A}$ pre-culture was prepared by adding a single colony of $E$. coli to $300 \mathrm{ml} \mathrm{LB}$ medium and

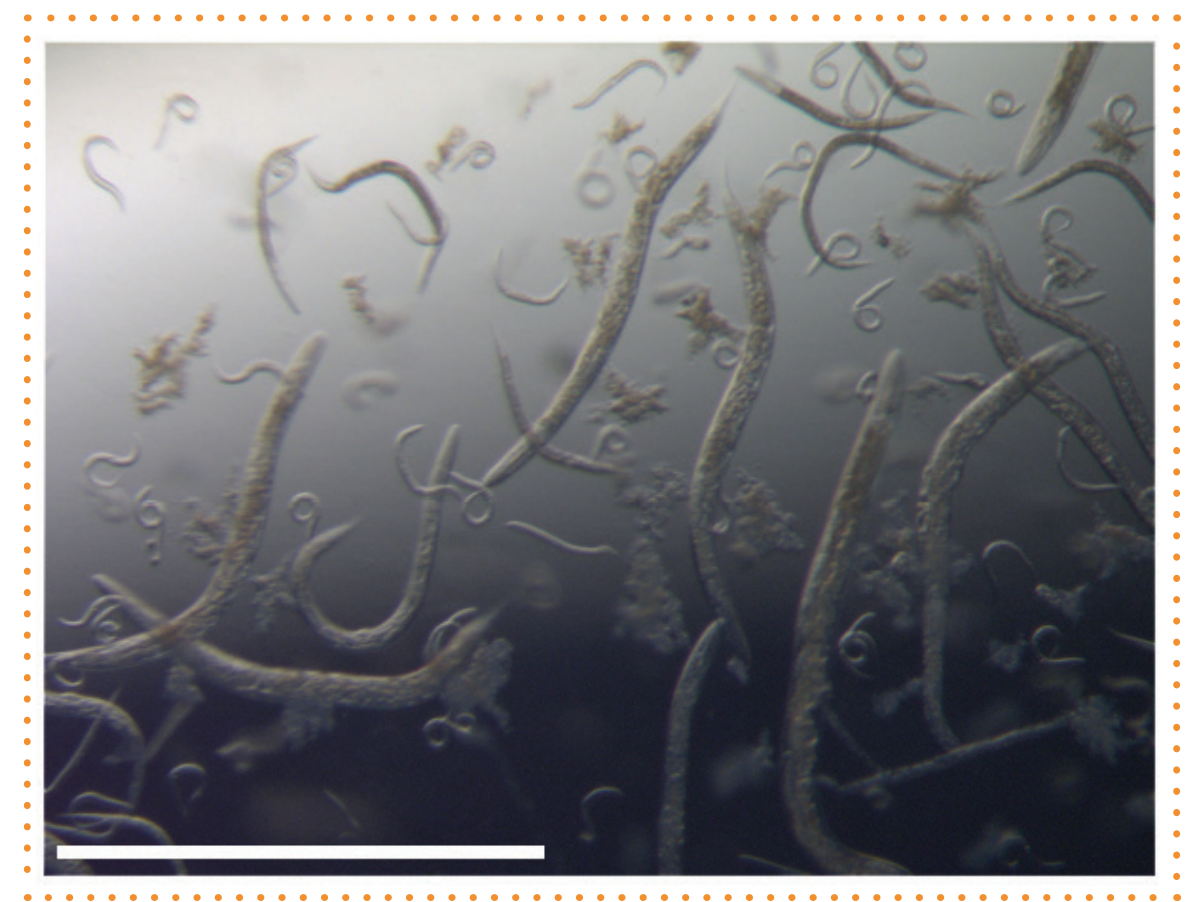

Figure 4. A representative image of an aliquot $(10 \mu \mathrm{l})$ from the bioreactor at $190 \mathrm{~h}$. At this point, there are approximately 10,000 worms $/ \mathrm{ml}$. It is striking that the media is relatively clear, indicating a lack of debris. Worms are visible at all stages and have a healthy morphology. Scale bar: $1 \mathrm{~mm}$.

grown overnight at $37^{\circ} \mathrm{C}$. Batch medium is prepared by sterilizing the carbon and nitrogen source (Table 1) separately to prevent Maillard reaction and precipitation. The sterile batch medium compounds are combined in a 7-I bioreactor (Table 1). Next, the bioreactor is inoculated with the $E$. coli pre-culture and left to run for $\sim 8 \mathrm{~h}$ (Table 2 ). At this point, the batch phase is completed, which can be observed as an increase in DO, and due to the lack of food available for the $E$. coli oxygen is consequently not required. When the batch phase is completed, the bioreactor is cooled to $10^{\circ} \mathrm{C}$ to keep the culture stable as feed for C. elegans and, by cooling, the $E$. coli does not need to be fed to remain viable.

\section{C. elegans cultivation}

A pre-culture of wild-type $C$. elegans (strain N2) is prepared by adding 30 L4 staged animals into $500 \mathrm{ml}$ of S-complete plus $50 \mathrm{ml} E$. coli culture. The pre-culture is left for 4 days at $20^{\circ} \mathrm{C}$ and $150 \mathrm{rpm}$.

C. elegans batch medium is prepared in a new 7-l bioreactor (Table 1). The bioreactor is inoculated with $100 \mathrm{ml}$ of the C. elegans pre-culture $(\sim 21 \mathrm{worms} / \mathrm{ml}$, calculated as the average of 10 aliquots of $20 \mu \mathrm{l}$ taken from a 1-ml sample from the
Erlenmeyer) and $15 \mathrm{ml}$ E. coli HB101 from the first bioreactor to start the cultivation and left to run. The addition of $E$. coli is achieved by running the feed pump. The pump speed should be calibrated, but is optimal at $200 \mathrm{ml} / \mathrm{h}$ with $100 \%$ pump speed. The DO should now drop, and may even hit $0 \%$, but this low concentration of oxygen is not harmful for $C$. elegans. The feed program can now be turned on via the Biocommand software, so when the DO increases to $20 \%$, the feed pump is turned on to add $E$. coli. Overfeeding is prevented by switching off the pump when the DO drops below $20 \%$. The cultivation is now left until the desired amount of $C$. elegans is reached.

\section{Harvesting and yield}

The bioreactor started with 21 worms $/ \mathrm{ml}$ in a total volume of 1 I. Over time, the addition of E. coli increases the total volume of the bioreactor and while this changes the concentration of the ingredients, we find that there are no noticeable adverse effects. However, it can be beneficial to add cholesterol when the worms do not appear to be developing. In this cultivation, cholesterol was added twice (Figure 3). In this experiment, we continued the bioreactor for a total of $270 \mathrm{~h}$, when 


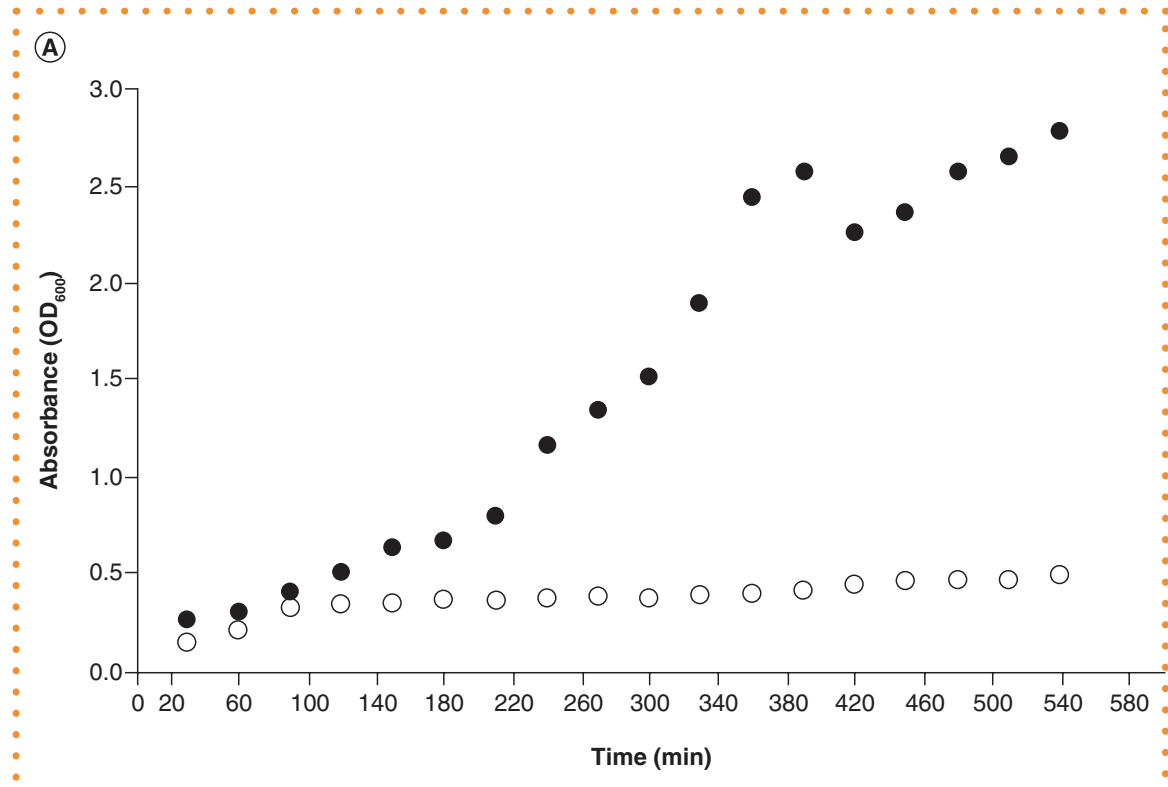

(B)

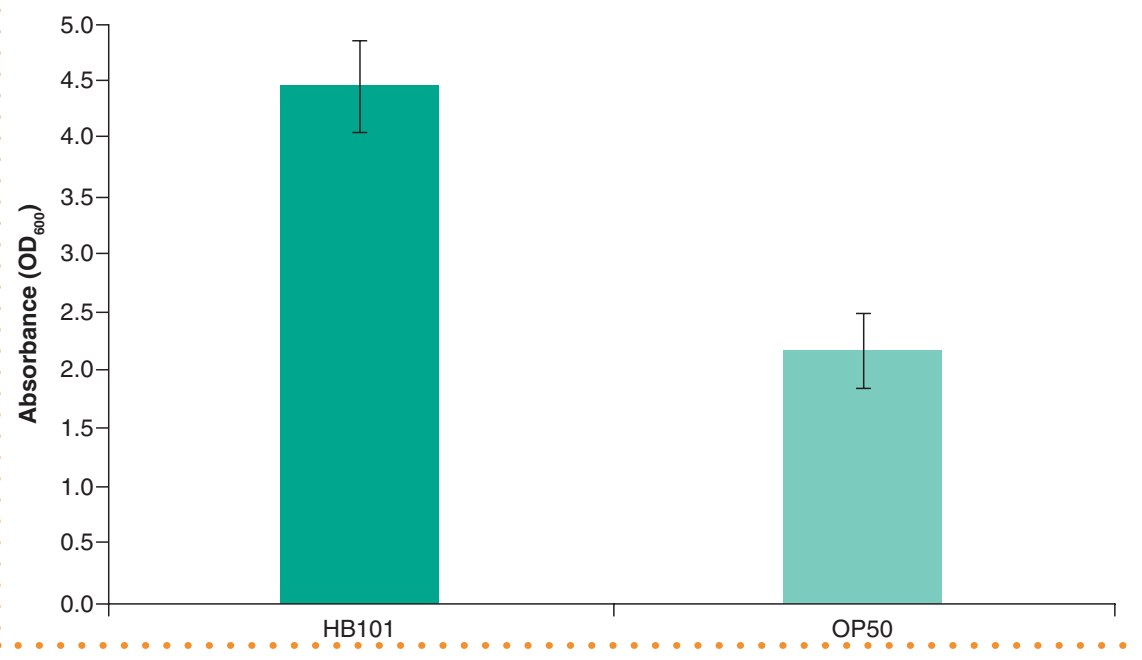

Figure 5. Growth curves comparing growth characteristics of Escherichia coli strains OP50 and HB101 in a 2-I bioreactor. Growth of HB101 (preferred bacterial food source for liquid culture) and OP50 (preferred food source for solid media) in a 2-I bioreactor using the same growth conditions and bioreactor setup were compared. (A) OP50 (open circles) has a slower growth compared with HB101 (closed circles) for the duration of the $540 \mathrm{~min}(9 \mathrm{~h})$ cultivation. Both strains of $E$. coli enter stationary phase between 3 and $8 \mathrm{~h}$. (B) Cultivation after $24 \mathrm{~h}$. The OD600 raises to 2.17 for OP50 while that for $\mathrm{HB} 101$ is at 4.47. Error bars are standard deviation.

- the yield was 24,000 worms $/ \mathrm{ml}$ in a total volume of $4.5 \mathrm{I}$. At each time point, the number of worms in the bioreactor was calculated as the average of 3-7 aliquots of $10 \mu$ l taken from a 5 - $\mathrm{ml} \mathrm{sample} \mathrm{from} \mathrm{the} \mathrm{bioreactor} \mathrm{and} \mathrm{scaled}$ up to the volume in the bioreactor at that time point. In all cases, the worms were developing as expected, displaying normal morphology and movement. The liquid medium did not contain debris (Figure 4), which means that after collecting the solution, worms could be pelleted by gravity. The $C$. elegans cultures

were constantly monitored, did not appear to be showing signs of stress and were not starved during the cultivation. Animals were healthy, displaying the thrashing movement expected from worms grown in liquid culture with no Dauer larvae present (Figure 4). Worms in the bioreactor developed normally as shown by the sigmoidal growth curve. To verify the health of the worms, aliquots were taken throughout the cultivation and left to develop on solid plates, where animals developed as expected.
It has to be noted that after approximately $250 \mathrm{~h}$, growth saturation is reached, at which stage most worms are adult hermaphrodites. If a mixed population with a good representation of all stages is required, we recommend harvesting cultures after 170-220 h. For instance, after $190 \mathrm{~h}$ the culture contains approximately $30 \%$ eggs and $50 \%$ larvae (Figure 4).

The protocol described here outlines an approach for cultivating large numbers of $C$. elegans. It is easily scalable and the two-bioreactor system can also be separated to be used for a one-bioreactor approach. In this setting $E$. coli are grown first according to the described protocol, harvested in a separate flask and fed to C. elegans via a pump on the flask. It is important to keep the $E$. coli cooled to $4-10^{\circ} \mathrm{C}$ to ensure it will not be metabolically active. In our experience, this has no effect on the quality of the bacteria. The $E$. coli is resuspended and fully mixed using the agitation of the bioreactor prior to using as a $C$. elegans food source. The yield obtained with this method is comparable to that of previously described protocols in which approximately 30,000 worms $/ \mathrm{ml}$ were collected [4]. However, while these worms were from 150-I cultures, we obtain the same yield from a 7-I bioreactor, which is more commonly available to research laboratories.

Alternative strains of $E$. coli can be used as $C$. elegans food source; however, $\mathrm{HB} 101$ is the recommended bacterial food source in liquid cultures of $C$. elegans $[12,13]$. If alternative bacteria are required, the cultivation conditions may need to be adjusted. We have found that OP50, the bacterial food source used for growing worms on solid media, has slower growth and consequently lower yields under the same conditions as HB101 (Figure 5A). For HB101, an 8-h cultivation is sufficient to generate enough food for a complete bioreactor run. However, as OP50 does not grow optimally, as expected [14], a longer cultivation is required to increase the yield (Figure 5B). Similarly, for $C$. elegans strains different from wild-type N2, altered culture conditions might also apply and temperature is an essential parameter due to the temperature sensitivity of many strains. In this bioreactor set-up changing the temperature is very simple via the cooling blanket and internal cooling coil. 
Thus, the relatively little effort required for this protocol, combined with system flexibility, provides a labor-friendly technology to reliably and reproducibly produce a high yield of healthy $C$. elegans.

\section{AUTHOR CONTRIBUTIONS}

The experiments were designed by $\mathrm{RH}, \mathrm{SH}$ and SP. BV performed the bioreactor experiments. $\mathrm{RH}, \mathrm{SH}$ and $\mathrm{BV}$ analyzed the data. The manuscript was prepared by $\mathrm{RH}, \mathrm{SH}$ and SP.

\section{FINANCIAL \& COMPETING INTERESTS DISCLOSURE}

This work was supported by the RAAKElegant! Project, funded by SiA 2014-0107PRO and the German Research Foundation (DFG: FOR 2149/P02). The authors have no other relevant affiliations or financial involvement with any organization or entity with a financial interest in or financial conflict with the subject matter or materials discussed in the manuscript apart from those disclosed.

No writing assistance was utilized in the production of this manuscript.

\section{ACKNOWLEDGMENTS}

The authors would like to thank Nil van $\mathrm{Hal}$ (Han University of Applied Sciences, The Netherlands) for his help with the bacterial cultivation. Preliminary work was supported by Antje Müller (Leipzig University, Germany), Eric van de Zilver and Rick van de Vondervoort (both at HAN University of Applied Sciences, The Netherlands). Caenorhabditis elegans and bacterial strains were provided by the CGC, which is funded by NIH Office of Research Infrastructure Programs (P40 OD010440).

\section{ETHICAL CONDUCT OF RESEARCH}

The authors state that they have obtained appropriate institutional review board approval or have followed the principles outlined in the Declaration of Helsinki for all human or animal experimental investigations.

\section{OPEN ACCESS}

This work is licensed under the Attribution-NonCommercial-NoDerivatives 4.0 Unported License. To view a copy of this license, visit http://creativecommons.org/ licenses/by-nc-nd/4.0/

\section{REFERENCES}

Papers of special note have been highlighted as: - of interest; $\cdot$ of considerable interest

1. Bandarankayke AD, Almo SC. Recent advances in mammalian protein production. FEBS Letters 588(2), 253-260 (2014).

2. Hellwig S, Drossard J, Twyman RM, Fischer R. Plant cell cultures for the production of recombinant proteins. Nature Biotechnol. 22, 1415-1422 (2004).

3. Li H, Wang Y, Xu A, Jin S, Wu D. Large-scale production purification and bioactivity assay of recombinant human interleukin-6 in the methylotrophic yeast Pichia pastoris. FEMS Yeast Res. 11(2), 160-167 (2011).

4. Gbewonyo $\mathrm{K}$, Roher $\mathrm{SP}$, Lister $\mathrm{L}$ et al. Large scale cultivation of the free living nematode Caenorhabditis elegans. Bio/Technology 12, 51-54 (1994).

.. This publication provides a protocol to produce $500 \mathrm{~g}$ of Caenorhabditis elegans from a 150-I bioreactor.

5. Gbewonyo K, Rohrer SP, Buckland BC. Bioreactor cultivation of the nematode Caenorhabditis elegans: large scale production of biologically active drug receptors for pharmaceutical research. Biotechno Genet. Eng. Rev. 14, 37-49 (1997).

6. Ehlers RU. Mass production of entomopathogenic nematodes for plant protection. Appl. Microbiol. Biotechnol. 56(5-6), 623-33 (2001).

- Provides information on dealing with common problems of liquid culturing to produce large volumes of matodes in bioreactors.

7. Fabian TJ, Johnson TE. Production of age-syncronous mass cultures of $C$. elegans. J. Gerontol. 49(4), B145B156 (1994).

-. Production of $C$. elegans on a large scale are described using wild-type and mutant strains.

8. Lewis JA, Fleming JT. Basic culture methods. Methods Cell Biol. 48, 3-29 (1995).

- The basic culturing of $C$. elegans in both small- and large-scale liquid media is described. The authors also include some information on the use of fermentation technology to scale up production.

9. Stretton AOW, Johnson CD, Anderson P. Buckets of worms. Worm Breeders Gazette 8(2), 56 (1984).

10. Shuler ML, Kargi F. Bioprocess Engineering: Basic Concepts (2nd Ed.). Prentice Hall, Upper Saddle River (2002)

11. Doran P. Bioprocess Engineering Principles. Elsevie Academic Press, 127-217 (1995).

12. Brooks KK, Liang $B$, Watts JL. The influence of bacterial diet on fat storage in C. elegans. PLoS One 4(10), e7545 (2009).

13. MacNeil LT, Watson E, Arda HE, Zhu LJ, Walhout AJ. Diet-induced developmental acceleration independent of TOR and insulin in C. elegans. Cell 153(1), 240-252 (2013)

14. Clark LC, Hodgkin J. Commensals, probiotics and pathogens in the Caenorhabditis elegans model. Cell. Microbiol. 16(1), 27-38 (2014). 